\title{
Actualización cartográfica del yacimiento arqueológico de Copán Ruinas, Honduras en el año 2016
}

\author{
Jessica Gabriela Villatoro Escobar \\ Eduardo Moreno Segura
}

\section{Resumen}

El objetivo de este trabajo fue actualizar la cartografía del Yacimiento Arqueológico de Copán Ruinas a un nivel más preciso de lo que existe en la actualidad. El proceso se llevó a cabo siguiendo una metodología descriptiva con enfoque cualitativo que consistió en el uso de Sistemas de Posicionamiento Global "GPS y DRON", utilizados en campo para apreciar el terreno con mayor claridad. El empleo de Sistemas de Información Geográfica fue de vital importancia para el agrupamiento, delimitación y digitalización de datos geográficos organizados en capas temáticas. Los resultados se presentan de forma descriptiva en diferentes momentos, uno donde se obtuvo un orto mosaico y modelo digital de superficie, otro donde se obtuvo un mapa de sombras y estructuras arqueológicas, finalmente el mapa de sitio Maya "Copán" actualizado, donde se aprecia el área de estudio con mayor claridad y precisión gracias a la resolución empleada. Se concluye que la actualización cartográfica, en especial, la del Yacimiento de Copán Ruinas es de mucho interés para entes gubernamentales y no gubernamentales, así como, universidades y otros que desean generar otros estudios, teniendo como insumo principal información de primera mano que facilite otros procesos como: la distribución de recursos físicos, naturales y económicos (inversiones nacionales y extranjeras), todo en torno al buen manejo del sitio arqueológico que representa riqueza para el territorio hondureño.

Palabras Clave: Sitio Arqueológico, Mosaico, Acrópolis, parque arqueológico.

\section{Abstract}

The objective was to update the mapping of the Archaeological Site of Copán Ruins at a more precise level than it exists today. The process was carried out following 
a descriptive methodology with a qualitative approach that consisted in the use of Global Positioning Systems "GPS and DRON", used in the field to appreciate the terrain with greater clarity. The use of Geographic Information Systems was of vital importance for the grouping, delimitation and digitization of geographic data organized in thematic layers. The results are presented descriptively at different times, one where a mosaic orthographic and digital surface model was obtained, another where a map of shadows and archaeological structures was obtained, finally the updated "Copán" Maya site map, where we can see the area of study with greater clarity and precision thanks to the resolution used. It is concluded that the cartographic update, especially the Copán Ruins Reservoir is of great interest to governmental and non-governmental entities, as well as universities and others that wish to generate other studies, having as main input first-hand information to facilitate others Processes such as the distribution of physical, natural and economic resources (national and foreign investments), all about the good management of the archaeological site that represents wealth for the Honduran Territory.

Keywords: Archaeological Site, Mosaic, Acropolis, archaeological park.

Jessica Gabriela Villatoro Escobar, (jessicavillatoro77@gmail.com) Universidad Nacional Autónoma de Honduras, Facultad de Ciencias Espaciales, Departamento de Ciencia y Tecnologías de la Información Geográfica.

Eduardo Moreno Segura, (eddmorse7@gmail.com) Universidad Nacional Autónoma de Honduras, Facultad de Ciencias Espaciales, Departamento de Ciencia y Tecnologías de la Información Geográfica.

Fecha de recepción: 14 de febrero de 2017. Fecha de aceptación: 14 de agosto de 2017. 


\section{INTRODUCCIÓN}

El Municipio de Copán Ruinas se ubica en Centro América, al Occidente del Departamento de Copán, República de Honduras. Colinda al norte y al oeste con la República de Guatemala, al sur con el Departamento de Ocotepeque y al este con los Municipios de Santa Rita y Cabañas, Departamento de Copán (ver figura 1).

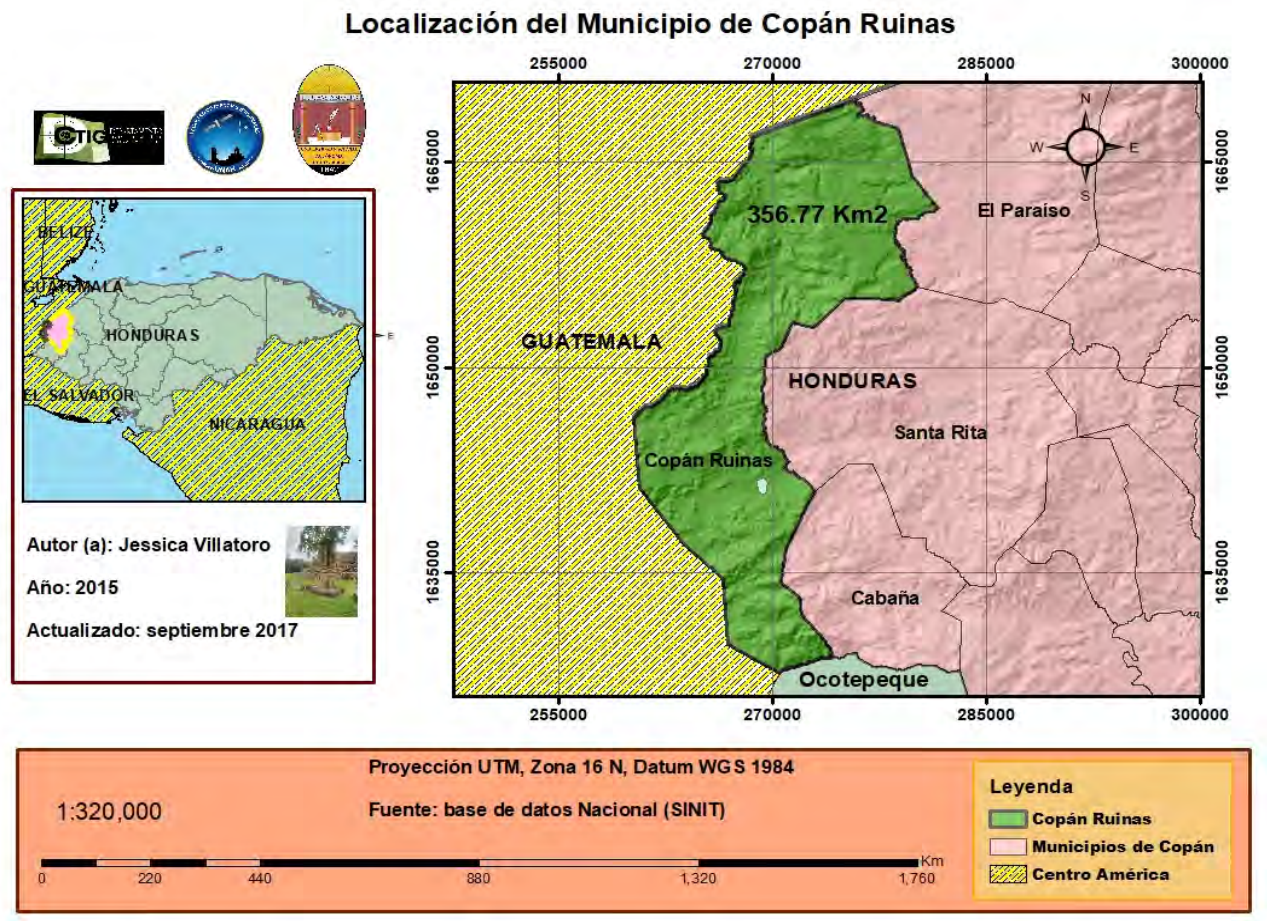

Figura 1. Localización del Municipio de Copán Ruinas, fuente: elaboración propia.

La topografía del Municipio es irregular, constituida por montañas y cerros, debido a que cruza un ramal de la Sierra del Gallinero, que es parte de la sierra del Merendón, donde se alcanzan alturas promedio de 1,200 m.s.n.m. (USAID, s.f.).

Dentro del Municipio de Copán Ruinas se encuentra, el Parque Arqueológico Maya Copán, a una elevación que alcanza $620 \mathrm{msnm}$, este representa un lugar atractivo y con mucha fuente de información valiosa sobre la antigua civilización Maya (Agurcia, 2004). 
Con el transcurrir de los años, son varios los instrumentos jurídicos que protegen el Sitio Maya de Copán, algunos de ellos son: la declaratoria de "Zona Nacional de Antigüedades" (1843) hasta el Acuerdo Presidencial No. 185 (junio 1982), que establece el Monumento Nacional de Copán (IHAH, 2013).

Las Ruinas de Copán fueron declaradas patrimonio de la humanidad por la UNESCO en 1980. La pequeña ciudad de Copán Ruinas, alberga el sitio arqueológico que constituye un atractivo turístico adicional, por la excelente conservación de su casco colonial (CLACDS/INCAE, 2003).

Por otro lado, se sabe que: "en los últimos años, los Sistemas de Información Geográfica (SIG o GIS en inglés), se han convertido en una herramienta clave para el estudio y la interpretación del Patrimonio Histórico. La posibilidad de documentar todos los datos espaciales del entorno de un yacimiento, así como poder hacer consultas que van más allá de la simple observación visual, convierten a los SIG en un recurso clave para el desarrollo actual de la investigación arqueológica" (CIEMAD, s.f.).

El proyecto descrito en este artículo es relevante por la dimensión geográfica presente en gran parte de los ámbitos de trabajo de la Arqueología vinculados con su gestión y protección. En este caso se hace necesaria una correcta gestión de la información, por lo que se procedió a obtener una actualización cartográfica del sitio Arqueológico Maya Copán (parque y acrópolis), obteniendo mucha más precisión de medición y exactitud, lo que es de mucha importancia para otros análisis complejos, ya que representa la base de otras líneas de investigación.

\section{METODOLOGÍA}

Consistió en la utilización de una técnica de modelamiento espacial de la zona de Influencia del Sitio Maya de Copán Ruinas, más específicamente, del parque arqueológico y la acrópolis, este último considerado patrimonio mundial.

\section{Enfoque}

El enfoque del estudio es cualitativo ya que se obtuvo información mediante la recolección de campo levantada mediante el uso de Sistemas de Posicionamiento Global "GPS y DRON". En tal sentido se puede dar una apreciación descriptiva y actualizada del área de estudio de Copán Ruinas. 
Para realizar una precisa modelización del parque arqueológico se contemplaron datos actualizados, conformando la profundidad del estudio.

\section{Descripción de instrumentos y técnicas}

- GPS: fue empleado para tener una ubicación espacial precisa sobre el área de estudio, además para generar un inventario estandarizado útil para posteriores análisis.

- DRON EBee: es una herramienta tecnológica de vehículo aéreo no tripulado que permite tomar imágenes de terreno en alta resolución que con su software especializado permite generar ortomosaicos y modelos de elevación de terreno de alta precisión, en este proyecto fue posible utilizar esta tecnología gracias al apoyo del Instituto de Conservación Forestal ICF quienes tienen este equipo para monitoreo forestal.

- La imagen obtenida mediante un DRON, fue punto fundamental para este estudio, ya que se trabajó mediante vuelo de paso bajo obteniendo imágenes de 3 centímetros de resolución (Zona de la Acrópolis) y 10 centímetros de resolución, con un vuelo de paso medio en una cobertura de 1,951.194 hectáreas correspondiente a la zona de influencia del Sitio Maya Copán.

- La Cartografía obtenida que nos permitió apreciar el aspecto temático y realizar la descripción del área de estudio, así como la actualización y comparación de cartografía temática generada por estudios previos en Copán Ruinas.

\section{RESULTADOS Y DISCUSIÓN}

\section{Identificación, Orto mosaico y Modelo Digital de Superficie}

Además de identificar los límites del parque arqueológico de Copán, se consideró su condición como:

- Área protegida categoría monumento cultural mediante decreto legislativo 18582, así mismo se ubicó la acrópolis, utilizando cartografía oficial generada por el Instituto de Conservación y Desarrollo Forestal, Áreas protegidas y Vida Silvestre (ICF) e imágenes georreferenciadas de la zona, obtenidas mediante el programa de Google Earth y hojas cartográficas a escala 1:50,000, proyección UTM y datum WGS_1984_16N. 
- Dicho procedimiento fue útil para el posterior levantamiento de información de campo e hizo evidente la necesidad de actualizar la cartografía del Yacimiento Arqueológico de Copán Ruinas (parque arqueológico y acrópolis), mediante levantamiento con el DRON, debido a que ésta estaba generada para una visualización a nivel nacional lo cual se vio reflejada en la baja presión en el área de estudio.

- Los datos obtenidos en campo mediante GPS y DRON, fueron organizados, tratados y representados geográficamente por medio del programa especializado en SIG, llamado ArcGis 10.3. Además, fueron georreferenciados a un mismo sistema de coordenadas proyectadas: Proyección UTM, Datum WGS84 Zona $16 \mathrm{~N}$.

- Mediante el DRON se planificaron 5 vuelos obteniendo alrededor de 2,000 imágenes de alta resolución, las cuales permitieron generar dos ortomosaicos de la Zona de estudio, uno de 10 centímetros de resolución (tamaño de pixel) y otro de la zona de la acrópolis de 3 centímetros de resolución (ver Figuras 2 y 3$)$.

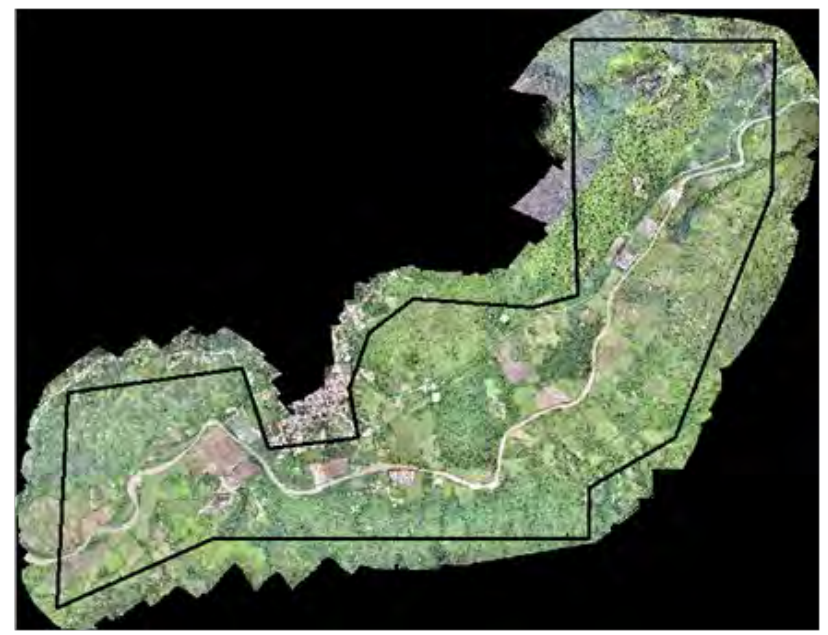

Figura 2. Ortomosaico de la zona de estudio, (10×10 cms. tamaño de pixel) de área de estudio (Zona de influencia Sitio Maya Copán). 


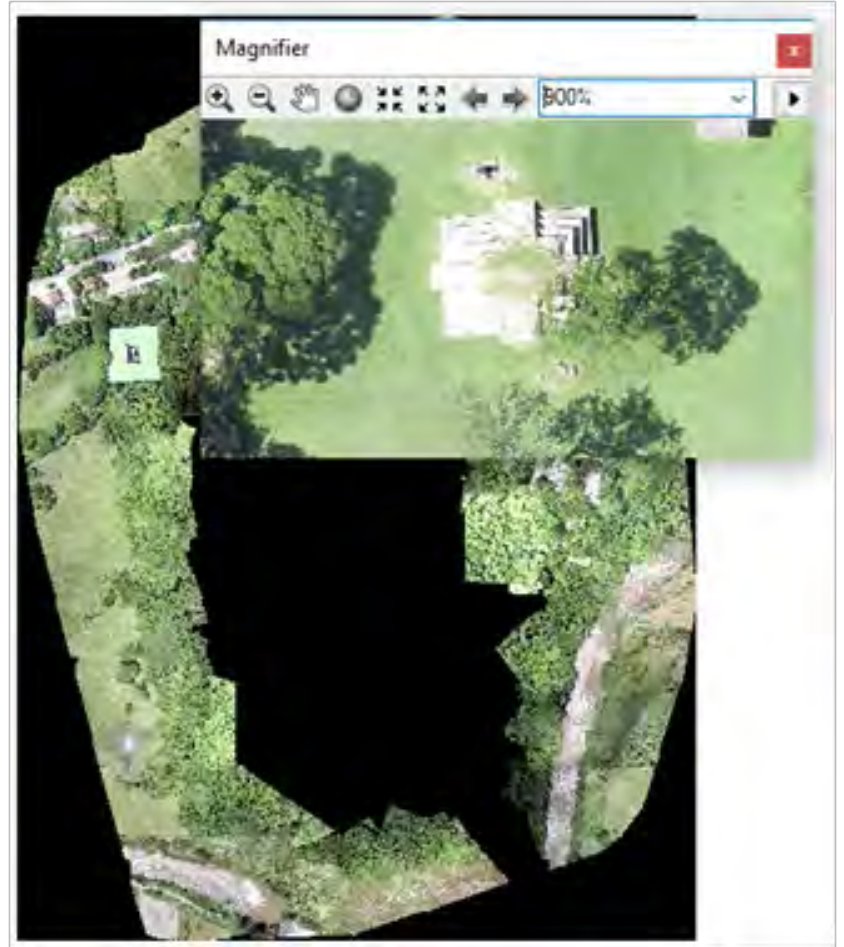

Figura 3.Ortomosaico (3×3 cms. tamaño de pixel) Zona de la acrópolis.

- Mediante los datos generados con el Dron fue posible generar un Modelo Digital de Superficie (MDS) el cual posteriormente fue procesado para obtener la planimetría (Curvas de nivel y mapa de Sombras) necesaria (ver Figura 4). 


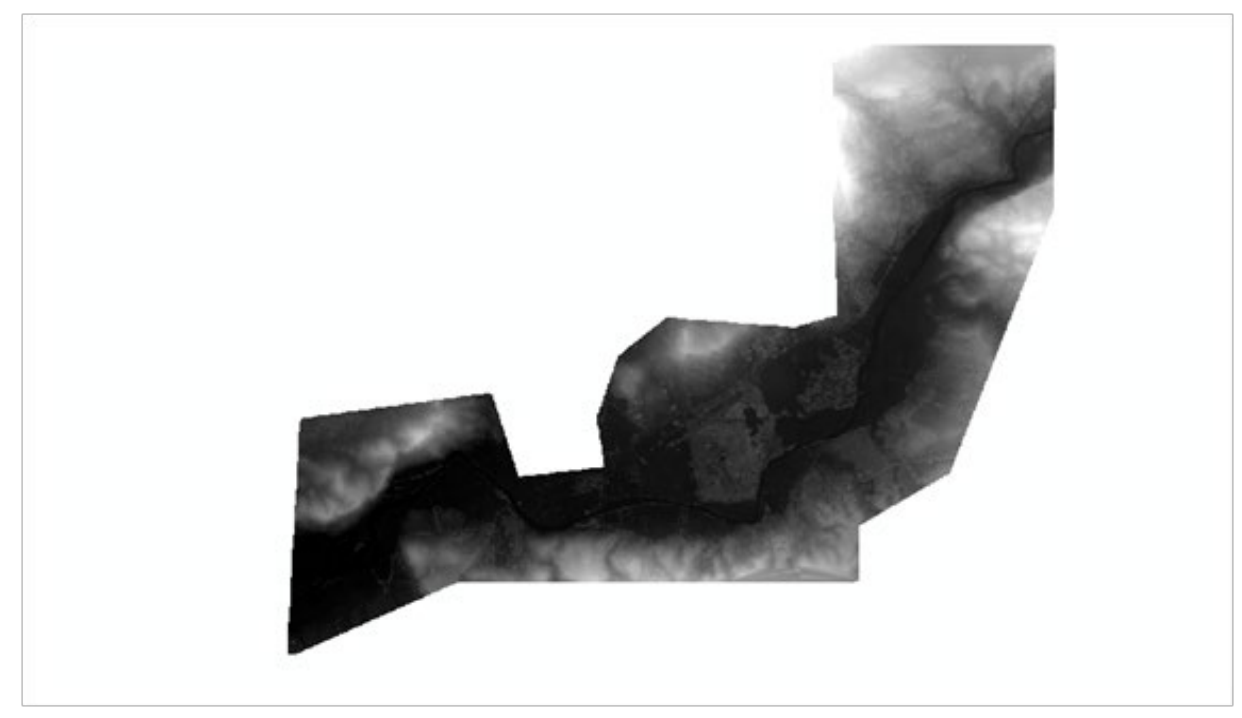

Figura 4. Modelo Digital de Superficie de área de estudio (Zona de influencia Sitio Maya Copán).

\section{Mapa de Sombras y estructuras arqueológicas}

Para obtener el mapa de Sombras y estructuras arqueológicas se procedió a digitalizar y procesar datos obtenidos del plan de manejo de Sitio Maya Copán 2005 y del Instituto Hondureño de Antropología e Historia. Acción que dio como resultado, la generaron de capas temáticas, útiles para la delimitación del Sitio Arqueológico Maya Copán y su Zona de Influencia.

En la Figura 5 se observa el límite del parque arqueológico de Copán, el límite de la propiedad de patrimonio Mundial (15.087 hectáreas), la zona de amortiguamiento de la propiedad de patrimonio Mundial (398.591 hectáreas) y el área de Influencia de la propiedad de patrimonio Mundial (1,951.194 hectáreas). 


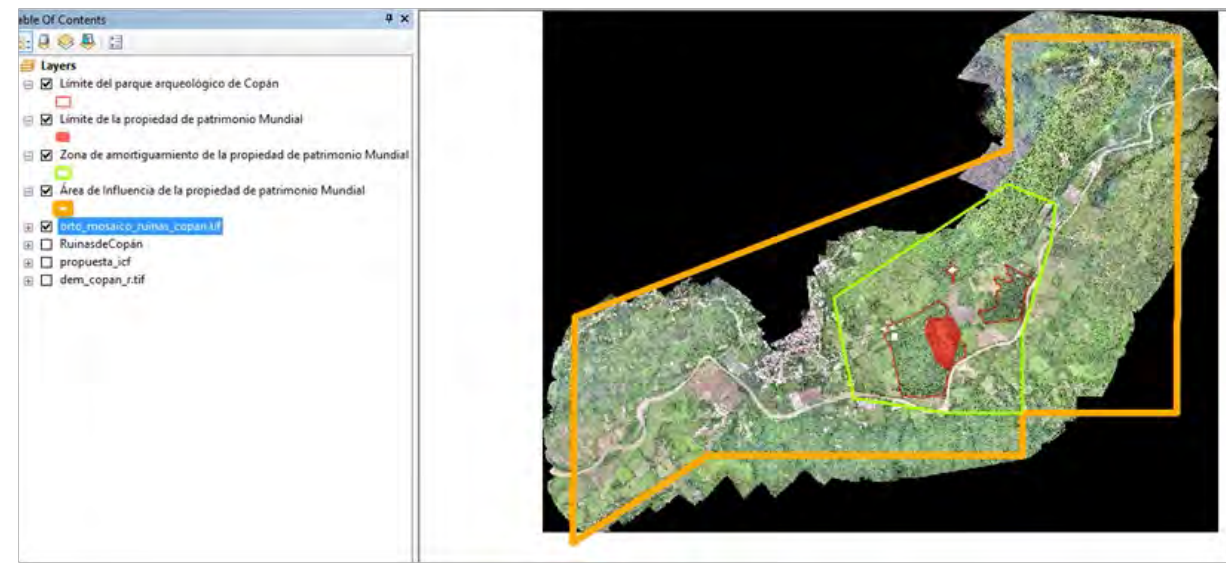

Figura 5. Capas Temáticas Sitio Maya Copán

Se digitalizaron las estructuras inventariadas por el Instituto Hondureño de Antropología e Historia, mismas que se transformaron a formato digital. Debido a que la información geográfica solo existía en formato análogo mediante mapas cartográficos. Para esto se escaneó, escaló, rotó y georreferenció dicha cartografía y se ajustó a la base cartográfica actualizada generada en este proyecto.

Mediante programas de Dibujo asistido por computadora como AUTOCAD y programas especializados en SIG, se obtuvieron las estructuras del Parque Arqueológico de Copán Ruinas (ver Figura 6).

\section{Mapa del Sitio Maya Copán Actualizado}

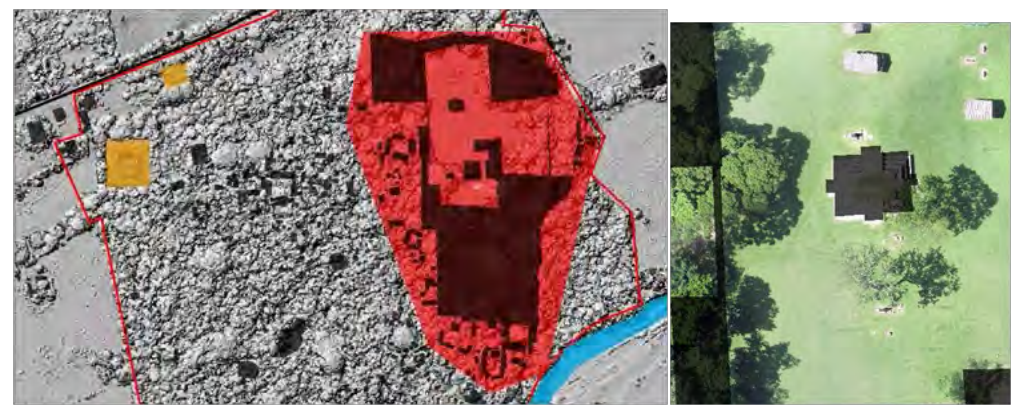

Figura 6. Estructuras arqueológicas área de la acrópolis de Copán. 
En el marco de los resultados prioritarios de este proyecto se generó el Mapa del Sitio Maya Copán (ver figura 7), bajo los estándares exigidos por UNESCO, ya que será tomado en cuenta como el posible mapa oficial actualizado ante el Instituto Hondureño de Antropología e Historia "UNESCO" de la propiedad de patrimonio mundial, para su publicación a nivel mundial, una vez revisado y aprobado por dicha contraparte o instituto.

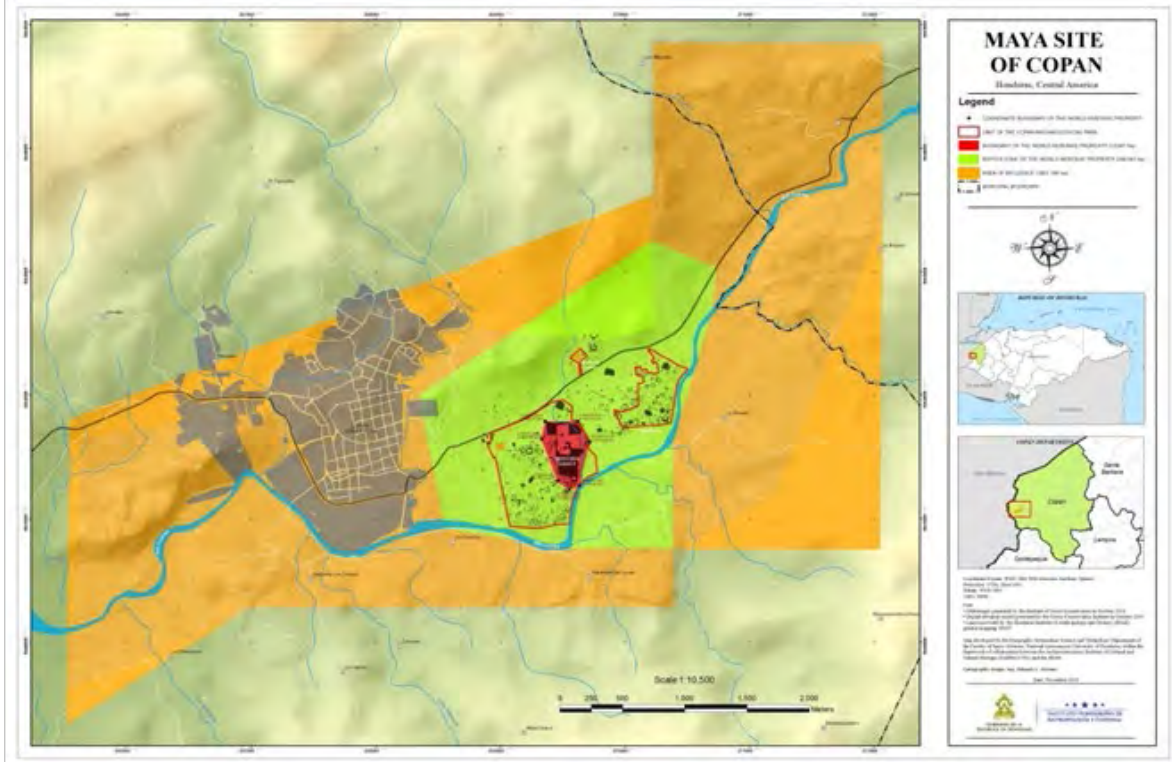

Figura 7. Mapa generado para UNESCO del sitio Maya Copán, actualizado en el 2016

\section{CONCLUSIONES}

1. Durante la investigación fue posible identificar la poca información georreferenciada en escala grande y oficialmente disponible del sitio Maya Copán (en formatos digitales editables), la cual solamente se encuentra en publicaciones escritas de investigaciones, planes de manejo o consultorías desarrolladas del Parque Arqueológico por instituciones y/o universidades extranjeras. En ese sentido fue necesario generar nuevamente información geográfica que aparecía solamente descrita en formatos impresos. 
2. De la comparación de distintas fuentes de información impresas fue necesaria la actualización del límite del parque arqueológico Maya Copán, ya que representa área de interés local, nacional e internacional, debido a que alberga importantes vestigios provenientes de los mayas.

3. La precisión y exactitud de los datos fue posible gracias al uso de alta tecnología (DRON), que con vuelos de mediana altura permitió obtener resolución de 10 centímetros para el Sitio Arqueológico Maya Copán y 3 centímetros para la Acrópolis que se encuentra dentro de los límites del Parque Arqueológico Maya Copán.

4. La implementación en este proyecto de tecnología DRON, ha demostrado una alta capacidad para el levantamiento de numerosa información actualizada del territorio a escalas muy grandes, que permiten el detalle y precisión de los datos derivados.

5. La cartografía actualizada sobre el Sitio Arqueológico Maya Copán viene a corregir problemas como la falta de información o datos crudos cartográficos en formato digital, que permitan la reutilización multifinalitaria de los mismos.

6. Con este proyecto se ha conformado la primera base de datos geográfica del Sitio Maya Copán con propósitos multifinalitarios de investigación en la UNAH, lo cual viene a corregir problemas como la falta de información o datos crudos cartográficos en formato digital, que permitan el acceso a información base para cualquier estudio territorial y análisis espacial en la Zona, así como, la reutilización multifinalitaria de los mismos.

7. Como uno de los productos más significativos de este proyecto se destaca el mapa oficial del Sitio Maya Copán actualizado al año 2016 y solicitado al Instituto Hondureño de Antropología e Historia (IHAH) por el Comité Patrimonio Mundial de UNESCO el cual pide proveer mapas con las delimitaciones exactas de la propiedad Patrimonio Mundial y su zona de amortiguamiento, basados en dos recomendaciones:

a) Cumplir con las decisiones del Comité Patrimonio Mundial, elaborando los mapas pedidos y regulaciones de la zona de amortiguamiento.

b) Colaborar con las autoridades locales para proteger el Sitio contra las presiones del desarrollo. 
8. Referente al estatus jurídico del Sitio Maya Copán, el Grupo Principal y sus alrededores fueron declarados propiedad del Estado en 1845. Los límites del Parque Nacional fueron fijados en Diciembre 1874, y la escritura del Parque fue trasferida al Instituto Hondureño de Antropología e Historia en mayo 1987. Ahora la situación jurídica del Parque Arqueológico de Copán es bastante compleja, con una parte propiedad del Estado, otra parte propiedad privada pero en custodia del Estado, y también una parte propiedad del Estado pero prestada a terratenientes.

Este proyecto toma vida gracias a la conformación del Instituto en Arqueoastronomía y Patrimonio Cultural y Natural (IARPACUNA), el cual surge como una alianza entre la UNAH y la Asociación Copán. El IARPACUNA en conjunto con la Asociación Copán gestionaron fondos ante la Copan Maya Foundation, permitiendo parte del financiamiento de este proyecto.

Se agradece al $\mathrm{IHAH}$ por brindar permisos para desarrollar investigaciones en el Sitio Arqueológico, así mismo, a la Universidad de Nebraska, por los datos proporcionados, al Instituto Nacional de Conservación y Desarrollo Forestal ICF por su apoyo y a Copan Maya Foundation por el financiamiento.

\section{BIBLIOGRAFÍA}

- Agurcia Fasquelle, R. (2004). Sobre el Sitio, Copán. Obtenido de Asociación Copán: http://asociacioncopan.org/sobre-el-sitio/

- CIEMAD, (s.f.). Uso de los SIG-GIS en Arqueología. Obtenido de Centro Internacional de Estudios Multimedia en Arqueología de Madrid: http://www.cursosarqueologia.com/cursos/software/sig-en-arqueologia

- CLACDS/INCAE, (2003). La Competitividad del Turismo en Honduras: Los Casos de la Ceiba y Las Ruinas de Copán. Alajuela, Costa Rica.

- $\quad$ IHAH, (2013). Sitio Maya de Copán, Plan de Manejo 2014-2020. Tegucigalpa.

- USAID, (s.f.). Estrategia participativa para el desarrollo integral del Municipio de Copán Ruinas. Copán. 